University of Nebraska - Lincoln

DigitalCommons@University of Nebraska - Lincoln

Gender Assignment for Directional Full-Duplex FDD Nodes in a

Multihop Wireless Network

Moein Parsinia

Qidi Peng

Sanjukta Bhowmick

John D. Matyjas

Sunil Kumar

Follow this and additional works at: https://digitalcommons.unl.edu/usafresearch

This Article is brought to you for free and open access by the U.S. Department of Defense at

DigitalCommons@University of Nebraska - Lincoln. It has been accepted for inclusion in U.S. Air Force Research by an authorized administrator of DigitalCommons@University of Nebraska - Lincoln. 


\title{
Gender Assignment for Directional Full-Duplex FDD Nodes in a Multihop Wireless Network
}

\author{
Moein Parsinia ${ }^{1}$, Qidi Peng ${ }^{2}$, Sanjukta Bhowmick ${ }^{3}$, John D. Matyjas ${ }^{4}$, \\ and Sunil Kumar ${ }^{5(凶)}$ \\ 1 Computational Science Research Center, San Diego State University, \\ San Diego, USA \\ mparsinia@mail.sdsu.edu \\ 2 Institute of Mathematical Sciences, Claremont Graduate University, \\ Claremont, CA, USA \\ qidi.peng@cgu .edu \\ 3 Computer Science Department, University of Nebraska at Omaha, Omaha, USA \\ sbhowmick@unomaha.edu \\ 4 Air Force Research Laboratory, Rome, NY, USA \\ john.matyjas@us.af .mil \\ 5 Electrical and Computer Engineering Department, San Diego State University, \\ San Diego, USA \\ skumar@mail.sdsu.edu
}

\begin{abstract}
The frequency-division duplex (FDD) nodes use two separate frequency bands (separated by a guard band) for transmission and reception, thus enabling the full-duplex (FD) communication. On the other hand, the use of directional FDD nodes in multihop wireless network offers the advantages of larger transmission range, better link reliability, and spatial reuse, resulting in a much higher throughput and superior interference mitigation. However, the multihop FDD communication partitions the nodes in two classes (or genders) wherein the nodes of the same class (or gender) in a neighborhood cannot communicate with each other. This can seriously impact the availability of neighboring nodes for communication, and lead to disconnected nodes (or regions) in the network. In this paper, an algorithm is presented to assign the appropriate genders to these nodes in a multi-hop network such that each node is able to communicate with its multiple 1-hop neighbors, located in different directions. Our simulation results demonstrate that approximately half of the neighbors of each node are of the opposite gender and they are distributed in different directions, thus enabling robust, multipath, and high throughput communication in the network.
\end{abstract}

Keywords: Frequency Division Duplex (FDD) - Full-duplex communication · Gender assignment - Frequency assignment • Directional antenna $\cdot$ Graph coloring $\cdot$ Multihop network 


\section{Introduction}

Multihop wireless networks are commonly used for the range extension and throughput improvement in ad hoc and peer-to-peer networks, with applications in sensor networks and emergency/disaster communication. Recently, the multihop communication (in the form of relaying) is also being used in wide-area cellular systems [1-3]. The multihop transmission can use time division duplex (TDD) or frequency division duplex (FDD) modes, both having their pros and cons. FDD uses two distinct frequency channels for transmitting and receiving the packets at the same time, while TDD operates on only one frequency channel where the transmission and reception of the packet(s) is interleaved in time. Since FDD does not need a guard time interval to separate the transmission from the reception, it is more efficient in wide area scenarios with long transmission links which introduce non-negligible signal propagation delays [4]. The fullduplex and/or half-duplex FDD modes have been proposed for the mobile radio systems like 3GPP LTE [3], GSM/GPRS [5], UMTS [6], WiMAX [7]. Recently, the FDD has also been proposed for $5 \mathrm{G}$ networks [8].

In an FDD system, the available frequency band $(\mathrm{F})$ is divided in two distinct bands (F1 and F2), with a guard band to separate them. The presence of guard band can allow a node to cancel the self-interference by sufficiently attenuating the received side-band power from its own transmission by using RF duplex filter. The node can therefore transmit the data on $\mathrm{F} 1$ and receive on $\mathrm{F} 2$ at the same time, leading to the full-duplex communication. In FDD, the F1 as well as F2 frequency bands can be grouped into blocks of contiguous channels. The available spectrum can thus be allocated to support the multiple users simultaneously on different bands. Since the transmissions on F1 and F2 bands in FDD systems are generally continuous, the receiver can feedback the most recent channel information to the transmitter. Thus, the delay is reduced for the channel information feedback, medium access control, and retransmission, which can enhance the system throughput [4].

Use of Directional Communication: The increasing use of high resolution multimedia applications by a rapidly increasing number of mobile users is making the wireless resources scarce. The proliferation of $5 \mathrm{G}$ devices would further aggravate this problem. As a result, the designers are considering the use of above $6 \mathrm{GHz}$ frequency bands (e.g., $12-18 \mathrm{GHz}, 28 \mathrm{GHz}, 60 \mathrm{GHz}$ and $72-73 \mathrm{GHz}$ bands), as larger RF spectrum bandwidth is available at these frequencies. However, the attenuation experienced by the signals (i.e., the path losses caused by the atmospheric path loss) is much higher at higher frequencies. So higher frequency links require more power. Use of directional antennas can be very helpful in realizing high data rate links as they increase the transmission range, besides offering spatial reuse $[9,10]$. The directional antennas are also more resistant to the interference as any unwanted signal which is not in the direction of the antenna beam has little impact on the node. Moreover, the use of multiple beam directional antennas (MBDA) in a wireless node allows the simultaneous transmission or reception on different beams using the same channel. As a result, 


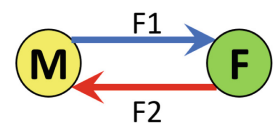

(a)

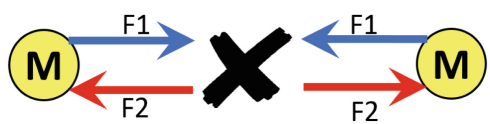

(b)

Fig. 1. FDD communication: (a) A node can communicate only with another node of opposite gender; (b) Two nodes of the same gender cannot communicate with each other.

the system capacity can be enhanced considerably. Fortunately, the size of these antennas also becomes more manageable at higher frequency bands.

In this paper, we consider a multihop wireless network consisting of the FDD nodes equipped with MBDA, which are operating in full-duplex mode. However, this partitions the nodes available in the network in two classes (or genders) as described below. If node $\mathrm{A}$ uses $\mathrm{F} 1$ for signal transmission and F2 for reception (we denote it as the male node), it can establish a link to communicate with another node B only if the latter receives the signal at F1 and transmits at F2 (we denote it as the female node). Node A cannot communicate with another node of the same gender (i.e., male node) that also transmits at $\mathrm{F} 1$ and receives at F2 (see Fig. 1). This requires that the frequency bands F1 and F2 in FDD nodes be chosen carefully for the transmission and reception such that a node could effectively use its multiple beams to establish links with its multiple 1hop neighbors, in order to simultaneously communicate with them. We call this problem as the gender assignment of the node.

When multiple nodes are available in a 1-hop neighborhood of a given node, the gender should be assigned such that this node can establish communication links with multiple neighboring nodes spread in different directions. This can help in establishing the routes which pass through the nodes in different parts of the network while avoiding the congested network nodes and regions. In fact, the gender should be assigned such that each node can establish links with approximately half of its 1-hop neighbors in different directions. This strategy allows almost all the nodes in the graph to have communication links with nearly half of their 1-hop neighbors.

\section{Proposed Distributed Gender Assignment Algorithm}

We consider solving the gender assignment problem as a simple undirected graph coloring problem. Recall that a simple undirected graph $G=(V, E)$ consists of a set of vertices $V$ and a set of edges $E$. Each edge $e \in E$ connects two vertices $u, v \in V$. This edge is thus represented as $e=\{u, v\}$. For two vertices $u, v \in V$, $u$ is called a neighbor of $v$ if $u$ and $v$ are joined by an edge $e$ (i.e., $\{u, v\} \in E$ ). Throughout this paper, we use graph to mean simple undirected graph for short.

We can create a representative graph of the wireless network as follows: Each wireless node is represented as a vertex in the graph. Two vertices are joined if the corresponding nodes are within a specified distance $d$ of each other 
(i.e., within a 1-hop distance). Since the nodes are assigned any one of two genders (i.e. male or female), the connections can be viewed as a bipartite graph. Recall that in a bipartite graph $(V, E)$, the set of vertices $V$ consists of two disjoint groups $V_{1}$ and $V_{2}$, in the sense that each edge in $E$ only connects a vertex in $V_{1}$ to another vertex in $V_{2}$ [11]. In the representative wireless graph, the nodes of each gender fall into a group. Then the connections between the nodes that can communicate with each other form a bipartite graph. It should also be noted that we want each node to connect to approximately half of its 1-hop neighbors.

Based on these observations, we now formulate our problem as follows. Let the representative graph of the wireless nodes be $G=(V, E)$. Assume genders are assigned (two colors) to the vertices. Therefore for each vertex $v$, its neighbors are either of opposite or the same gender. We denote $N_{B}(v)$ to be all the neighbors of different gender from $v$, and $N_{C}(v)$ to be all the neighbors of the same gender as $v$. Our goal is to solve the following optimization problem: determining a gender assignment such that $\left|N_{B}(v)\right| \approx\left|N_{C}(v)\right|$ for each vertex $v \in V$ (where $|A|$ denotes number of elements in the set $A$ ). This will satisfy the requirement that each node has approximately equal number of neighbors from each gender.

Although this problem seems to be similar to extracting the maximal (in the sense that the number of vertices is maximized) bipartite subgraph from some graph, it has certain additional interesting aspects as discussed below. The problem of finding the maximal bipartite subgraph is NP-complete. Here our problem is different and more complicated, because our target is not finding the "largest" subgraph, but rather an almost equal division between the 1-hop neighbors of each node. Moreover, because the wireless connections of all the nodes may not be known globally, we have to do this assignment using local information, one vertex at a time.

We present our heuristic distributed gender assignment algorithm below which attempts to maintain a balance between the number of male (M) and female (F) nodes in each 1-hop neighborhood. Also note that the placement of the male and female nodes in the 1-hop neighborhood of any node, in terms of the direction from this node, should be almost evenly distributed. The advantage of our algorithm is that it "locally optimizes the solution", i.e., it makes sure that each node has almost equal number of neighbors with different genders in its 1-hop neighborhood.

Before presenting the pseudocodes of our algorithm, we make some notation conventions. For each node $v, g(v)$ denotes its gender assignment $(1=\mathrm{M}$ and $0=\mathrm{F})$. Then we note that $1-g(v)$ is the opposite gender assignment to $v$ 's. We also denote $\operatorname{deg}_{G}(v)$ to be the number of 1-hop neighbors of $v$ in the graph $G$. We call $G_{1}$ a complementary graph of $G_{2}$ in $G$, if $G_{1}, G_{2}$ have disjoint sets of vertices, disjoint sets of edges and $G=G_{1} \cup G_{2}$. Moreover, if the set of vertices of $G_{2}$ is $V_{2}$, we denote $G_{1}=G \backslash V_{2}$. Finally we use $\arg \max _{v \in V} \operatorname{deg}_{G}(v)$ to denote the set of vertices in $V$ having the largest number of neighbors in $G$ ( $V$ is not necessarily the set of vertices of $G$ ). As a main result, the pseudocodes of our heuristic distributed gender assignment algorithm is given below: 
Pseudocodes: Heuristic Distributed Gender Assignment Algorithm

1: INPUT: a visible subgraph $G=(V, E)$ of the global graph, with $V=\left\{v_{1}, \ldots, v_{n}\right\}$ and $E=\left\{e_{1}, \ldots, e_{m}\right\}$

2: Step 1: First Seed Node Selection and its Gender Assignment.

3: Randomly equally likely pick $v_{0}$ in $V$. Denote by $N_{0}$ the set of $v_{0}$ 's 1 -hop neighbors in $G$.

4: Randomly equally likely pick $v$ in $\arg \max _{u \in N_{0}} \operatorname{deg}_{G}(u) ; g(v) \leftarrow 1$;

5: Step 2: Gender Assignment in 1-hop Neighborhood of the First Seed.

6: Determine 1-hop neighborhood. Let $\left(v_{1}, \ldots, v_{p}\right)$ be all the neighbors of $v$ in clockwise order, starting from the North Direction.

7: $N \leftarrow\left(v_{1}, \ldots, v_{p}\right)$;

8: Assign genders to 1-hop neighborhood of $v$.

9: for $k$ in $\{1, \ldots, p\}$, do:

10: $\quad$ if $k$ is odd, do: $g\left(v_{k}\right) \leftarrow 0$; else do: $g\left(v_{k}\right) \leftarrow 1$;

11: end for

12: Step 3: Next Seed Node Selection.

13: Consider the complementary graph of unassigned nodes $G^{\prime}$.

14: $G^{\prime} \leftarrow G \backslash N$; randomly equally likely pick $v^{\prime}$ in arg $\max _{u \in N \cup\{v\}} \operatorname{deg}_{G^{\prime}}(u)$;

15: Step 4: General Gender Assignment in 1-hop Neighborhood (Phase 1).

16: Determine unassigned 1-hop neighborhood. Let $\left(v_{1}^{\prime}, \ldots, v_{q}^{\prime}\right)$ be all the unassigned neighbors of $v^{\prime}$ in clockwise order, starting from the North Direction.

17: $N^{\prime} \leftarrow\left(v_{1}^{\prime}, \ldots, v_{q}^{\prime}\right)$;

18: Assign genders to unassigned 1-hop neighborhood of $v^{\prime}$.

19: for $k$ in $\{1, \ldots, q\}$, do:

20: $\quad$ if $\left|N_{B}\left(v^{\prime}\right)\right|-\left|N_{C}\left(v^{\prime}\right)\right|>2$, do: $g\left(v_{k}^{\prime}\right) \leftarrow g\left(v^{\prime}\right)$;

21: $\quad$ else if $\left|N_{C}\left(v^{\prime}\right)\right|-\left|N_{B}\left(v^{\prime}\right)\right|>2$, do: $g\left(v_{k}^{\prime}\right) \leftarrow 1-g\left(v^{\prime}\right)$;

22: $\quad$ else do: $N_{k}^{\prime} \leftarrow$ "1-hop neighborhood of $v_{k}^{\prime}$ ";

23: $\quad$ if $\left|\left\{u \in N_{k}^{\prime}, g(u)=1\right\}\right|>\left|\left\{u \in N_{k}^{\prime}, g(u)=0\right\}\right|$, do: $g\left(v_{k}^{\prime}\right) \leftarrow 0$;

24: $\quad$ else if $\left|\left\{u \in N_{k}^{\prime}, g(u)=1\right\}\right|<\left|\left\{u \in N_{k}^{\prime}, g(u)=0\right\}\right|$, do: $g\left(v_{k}^{\prime}\right) \leftarrow 1$;

25:

26: end for

27: Step 5: General Gender Assignment in 1-hop Neighborhood (Phase 2).

28: for $k$ in $\{1, \ldots, q\}$, do:

29: $\quad$ if $v_{k}^{\prime}$ disconnects other 1-hop neighbors of $v^{\prime}$, do: $g\left(v_{k}^{\prime}\right) \leftarrow 1-g\left(v^{\prime}\right)$;

30: end for

31: Step 6: Run Steps 3-5 with updated $G$ until all the nodes are assigned genders.

32: OUTPUT: Graph $G$ with gender-assigned nodes.

The following example uses a connected graph with 16 nodes. We assume that the 1-hop neighbors of a node are known. The algorithm iteratively selects a seed node in each step, and assigns the gender to the seed node and its 1-hop neighboring nodes. The 1-hop neighbors of a node are shown connected with the node by a solid or broken link in the graphs in our examples. 


\section{First Seed Node Selection:}

The first seed node is selected from a group of nodes consisting of a randomly selected starting node and its 1-hop neighbors, as described below. The number of 1-hop neighbors (whose gender has not yet been assigned) of each node from the above group, is recorded and the node with the maximum number of unassigned 1-hop neighbors is selected as the first seed node. If more than one node has the maximum number of unassigned 1-hop neighbors, one of them is randomly selected as the first node. This seed node is assigned the "male" gender.

For example in Fig. 2a, node 5 is selected as the starting node. It has four 1-hop neighbors $(9,10,15,14)$. Among these five nodes, node 15 is selected as the first seed and assigned the male gender, because it the maximum number of seven unassigned 1-hop neighbors.

2. Gender Assignment in 1-hop Neighborhood of the First Seed:

Gender of the 1-hop nodes of first seed is assigned in an alternating order (e.g., $\mathrm{M}$ followed by $\mathrm{F}$, or $\mathrm{F}$ followed by $\mathrm{M}$ ) in the clockwise direction, starting from the node in the North direction, such that all the nodes in 1-hop neighborhood are equally (or nearly equally) divided in two genders. The first 1-hop node is assigned a gender opposite of the seed node. Two consecutive nodes in the clockwise order are assigned opposite genders. Note that our algorithm will work equally well if we select the anti-clockwise order and chose a node starting in a direction other than the North, as long as the same convention is followed.

In Fig. 2a, Node 15 is the first seed node and its 1-hop neighbors in the clockwise order starting from North are 3, 1, 4, 14, 5, 10 and 7 . The gender to

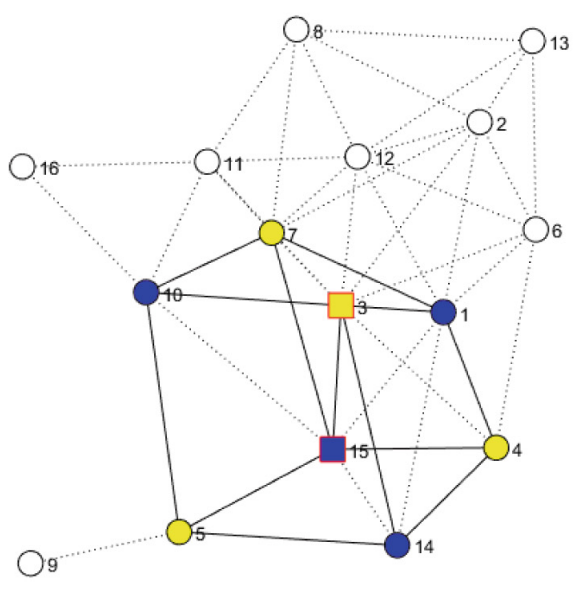

(a)

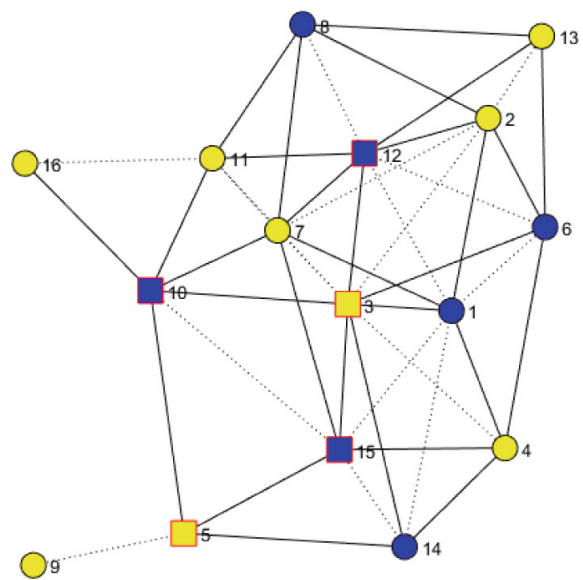

(b)

Fig. 2. (a) First seed selection for the neighborhood of node 5 and gender assignment in the neighborhood of node 15, (b) Gender assignment in phase 2 for the neighborhood of seed node 5 . Node 9 will change to a male gender to keep it connected. 
first node (node 3) is assigned as F (which is opposite of seed node) and the remaining nodes are assigned gender in an alternating order in the clockwise direction. The result of this assignment is shown in Fig. 2a.

3. Next Seed Selection:

The next seed is selected from the list of all the assigned nodes in the graph. The node which has the maximum number of the unassigned nodes in its 1-hop neighborhood is the candidate seed node. If more than one such nodes exist, the seed is randomly selected from the list of candidate seed nodes.

In Fig. 2a, node 3 is selected as the next seed (after seed 15) since it has the maximum number of four unassigned neighbors with nodes $[12,2,6,11]$.

4. General Gender Assignment in 1-hop Neighborhood (Phase 1):

A gender to each unassigned 1-hop neighbor node of the current seed is assigned in the clockwise order, by considering the following two criteria:

(i) The difference in the number of male and female nodes in the 1-hop neighborhood of the seed should not exceed a threshold (we use a threshold of 2 here), and ( $i i)$ The difference in the number of already-assigned male and female nodes in its 1-hop neighborhood, including the gender of the current node is minimized.

For example, in Fig. 2a, node 3 is the seed with nodes $[12,2,6,11]$ as its unassigned 1-hop neighbors in the clockwise order. For deciding the gender of the first node 12 , we first check the difference between the male and female nodes in the 1-hop neighborhood of seed node 3 , which is one. In the 1-hop neighborhood of node 12 , the nodes 3 and 7 are female and nodes 1 is the only male. Therefore, its gender is assigned as male which satisfies both the above criteria. For the next node 2, again we first check the difference between the male and female nodes in the 1-hop neighborhood of seed node 3 which in this case is three; so node 2 is assigned the female gender to minimize the difference between the male and female nodes in the neighborhood of seed node based on criteria (i).

Exception: If there are equal number of already-assigned male and female nodes in the 1-hop neighborhood of a given node, its gender is assigned as the opposite gender of the current seed.

\section{General Gender Assignment in 1-hop Neighborhood (Phase 2):}

After assigning gender to all the 1-hop neighbors of the current seed node in Phase 1, the connectivity of each of these nodes is checked. If any node is left unconnected, its gender is changed to the opposite gender of the current seed. For example, the node 9 in the neighborhood of seed node 5 in Fig. $2 \mathrm{~b}$ is left unconnected in Phase 1. Therefore, its gender will change to male, which is opposite gender of seed. Thus, node 9 will have one link available for communicating with its one hop neighbor (node 5 ).

6. The next seed is selected as discussed in Step 3 and gender assignment performed in its 1-hop neighborhood until all the nodes are assigned a gender. 


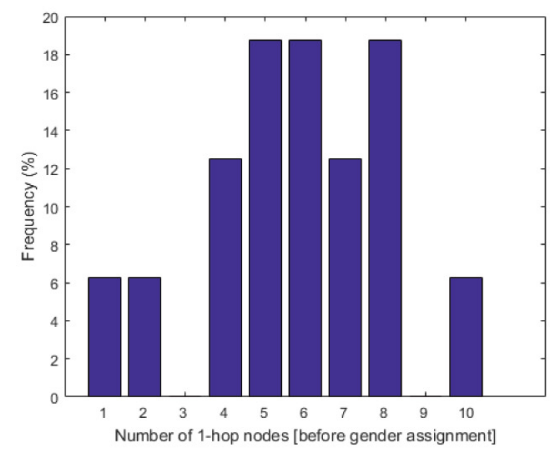

(a)

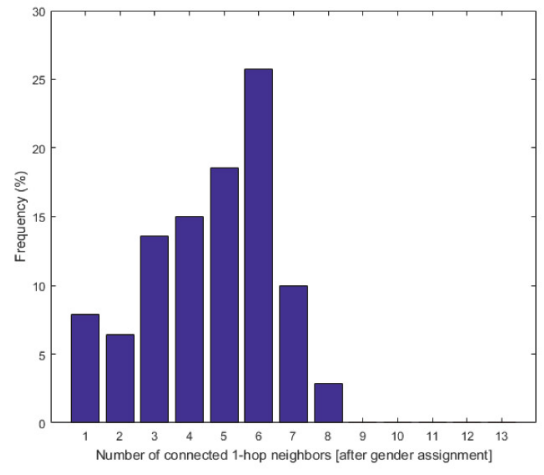

(b)

Fig. 3. (a) Histogram of the number of 1-hop neighbors before the gender assignment, (b) Histogram of the number of connected 1-hop neighbors after the gender assignment, for the random graph of 16 nodes shown in Fig. $2 \mathrm{~b}$.

\section{Performance Evaluation}

In this section, performance of the proposed gender assignment algorithm is evaluated for two different graphs. The first example is a random graph with 16 nodes (Fig. $2 \mathrm{~b}$ ) and the second example is a $5 \times 5$ grid graph. The results are average for 1000 runs with different starting nodes. The 1-hop neighbors of a node are determined such that their Euclidean distance from the node is below a threshold. A histogram representing the frequency of occurrence of the number of 1-hop neighbors for each node in the random graph of 16 nodes (before gender assignment) is shown in Fig. 3a. For example, an average of $12 \%$ of nodes have 4 nodes in their 1-hop neighborhood (before gender assignment). A node can communicate with other nodes of opposite gender in its 1-hop neighborhood. We call these nodes as the "connected 1-hop neighbors". Figure 3 b shows the histogram representing the frequency of occurrence of the connected 1-hop neighbors for each node in the graph, after the gender assignment. We observe that more than $90 \%$ nodes have at least 2 connected 1-hop neighbors, and about $85 \%$ nodes have 3 or more connected 1-hop neighbors. Figure 4 a shows the number of male and female nodes in the 1-hop neighborhood of each node of the graph. We observe that the 1-hop neighbors of a node are almost equally divided among the male and female genders for all the 16 nodes of the graph. A histogram of the difference between the number of male and female nodes in 1-hop neighborhood of each node of the graph is shown in Fig. 4b. This plot further verifies that the maximum difference between the number of male and female nodes is less than 2.2 in 1-hop neighborhood of each node.

A sample of the gender assignment output for a $5 \times 5$ grid graph is shown in Fig. 5a. Before the gender assignment in this graph, nine nodes had eight 1hop neighbors each, 12 edge nodes had five 1-hop neighbors each, and the four corner nodes had 3 neighbors each. After the gender assignment (see Fig. 5b), we 


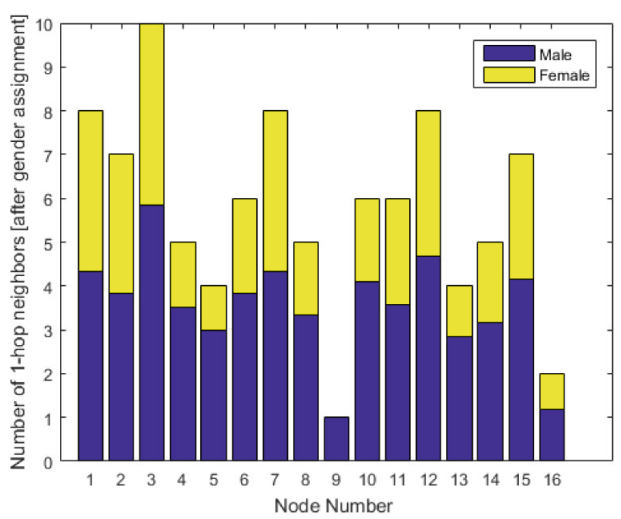

(a)

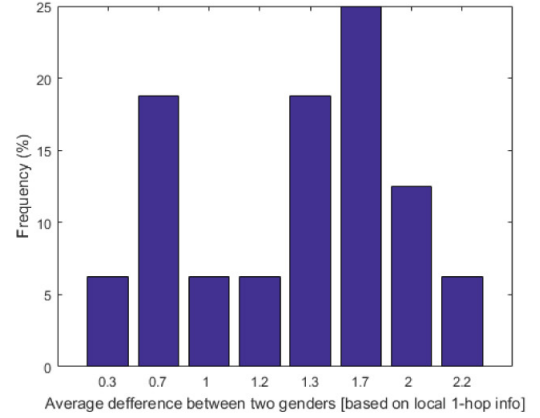

(b)

Fig. 4. (a) Gender distribution in the 1-hop neighborhood of each node, (b) Histogram of the difference between two genders among 1-hop neighbors, for the random graph of 16 nodes shown in Fig. $2 \mathrm{~b}$.

observe that all nodes have at least two connected 1-hop neighbor, and $80 \%$ nodes have three or more connected 1-hop neighbors. Figure 6a shows that the 1-hop neighbors of every node are almost equally divided among the male and female genders. Figure $6 \mathrm{~b}$ shows that the maximum difference between the number of male and female nodes in the 1-hop neighborhood of each node of the graph is less than 2.3 .

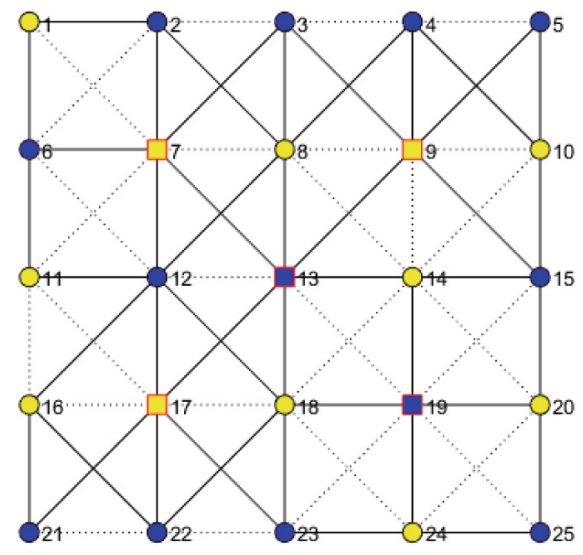

(a)

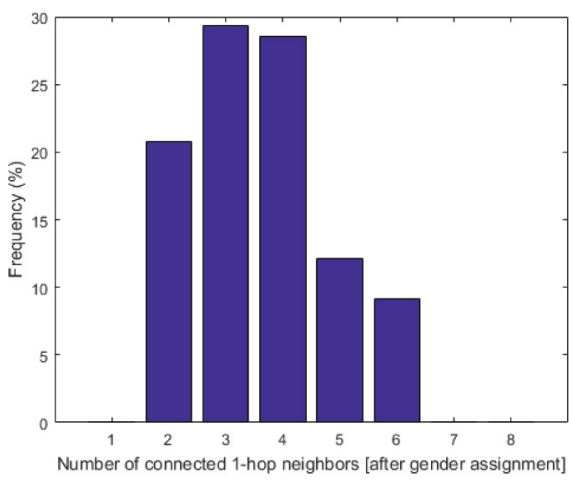

(b)

Fig. 5. (a) A sample gender assignment result, for the $5 \times 5$ grid graph, (b) Histogram of the number of connected 1-hop neighbors after gender assignment. 


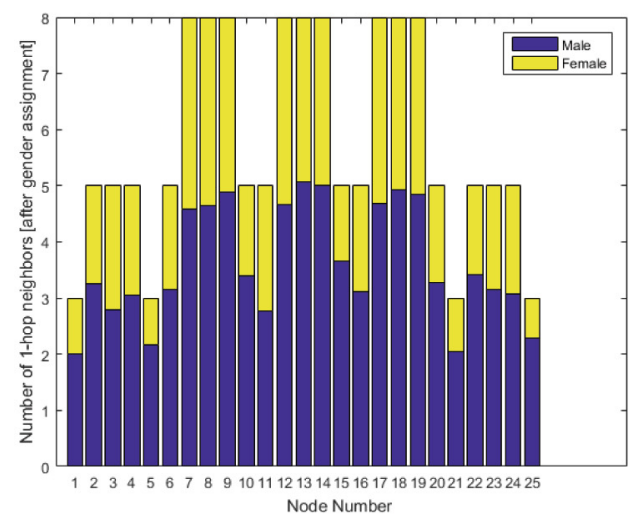

(a)

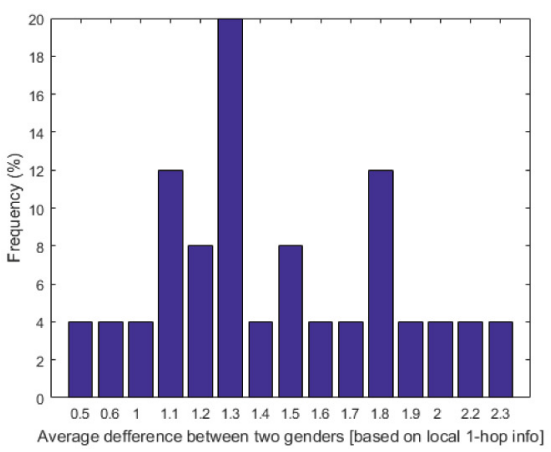

(b)

Fig. 6. (a) Gender distribution in the 1-hop neighborhood of each node, (b) Histogram of the difference between the nodes of two genders among 1-hop neighbors.

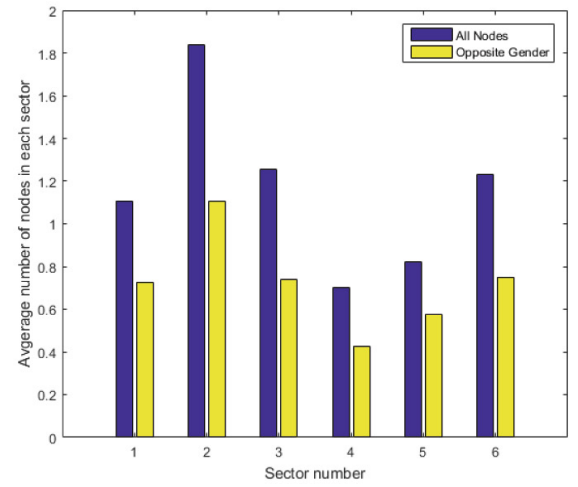

(a)

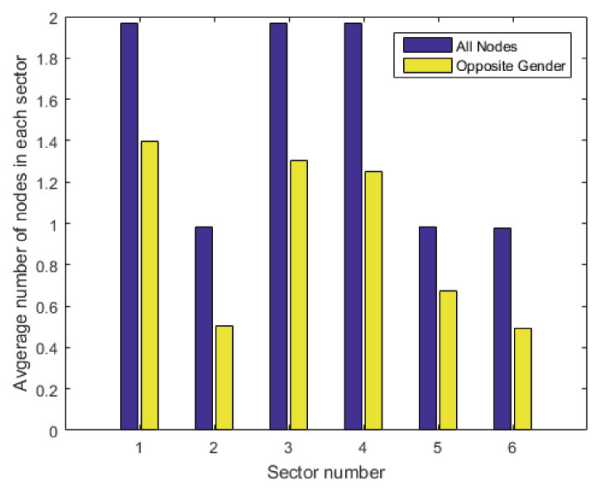

(b)

Fig. 7. Average distribution of genders in 1-hop neighborhood of all the seed nodes: (a) for the random graph in Fig. 2b, (b) for the grid graph in Fig. 5a. The results are average of 1000 runs.

Average distribution of nodes in different directions for both graphs is illustrated in Fig. 7. We have used six sectors of $60^{\circ}$ each in these plots, which cover all the directions. We observe that our gender assignment algorithm assigns nearly half the 1-hop nodes to opposite gender in each sector. The Y-axis of these plots shows fractional number because we have taken an average for all the seeds over 1000 runs.

Our algorithm thus assigns equal (or nearly equal) number of colors (genders) in every neighborhood for both graphs. After the gender assignment, there isn't any unconnected node in either graph and each node can communicate with approximately half of its neighbors in different directions. 


\section{Conclusion and Future Work}

We considered a multihop wireless network consisting of the directional FDD nodes equipped with MBDA, operating in full-duplex mode. Such networks offer the advantages of higher throughput, better link reliability, and superior interference mitigation. However, the multihop FD-FDD communication partitions the nodes in two classes (or genders) wherein the nodes of the same class (or gender) cannot communicate with each other. This can seriously impact the communication between neighboring nodes. Therefore, the gender (or frequency channel) of these nodes should be selected such that each node is able to simultaneously communicate with its multiple 1-hop neighbors, located in different directions.

A heuristic, distributed gender assignment algorithm was presented to assign the appropriate gender to these directional, FD-FDD nodes in a multi-hop network. Our simulation results demonstrated that our algorithm successfully assigned the gender to all the nodes in the network, such that each node could establish communication links with approximately half of its 1-hop neighbors in different directions. This arrangement can also help in establishing the robust and multi-path routes which pass through nodes in different parts of the network while avoiding the congested network nodes and regions. To the best of our knowledge, this is the first algorithm to extract a balanced bipartite graph, where the neighbors of the bipartite graph is roughly equal to the remaining neighbors in the graph. In addition to being an interesting and complex problem, this problem is solved using only local node information, and not the global knowledge of the network.

Acknowledgments and Disclaimer. This work was supported by U.S. Department of Defense under Grant No. FA8750-14-1-0075. Approved for public release; Distribution Unlimited: 88ABW-2016-3523, 18 July 2016. Any opinions, findings and conclusions or recommendations expressed in this material are those of the author(s) and do not necessarily reflect the views of DOD or U.S. government. The authors also acknowledge Drs. John Boyd and Kevin Chang, Cubic Corp. for technical discussions.

\section{References}

1. Otyakmaz, A., Walke, B.H.: Concurrent operation of half- and full-duplex terminals in future multi-hop FDD based cellular networks. In: International Conference on Wireless Communications Network and Mobile Computing, October 2008. 10. 1109/WiCom.2008.72

2. Pabst, R., et al.: Relay-based deployment concepts for wireless and mobile broadband radio. IEEE Commun. Mag. 44, 80-89 (2004)

3. Schoenen, R., et al.: On PHY and MAC performance of 3G-LTE in a multi-hop cellular environment. In: WiCom 2007, September 2007

4. Chan, P.W.C., Lo, E.S., et al.: The evolution path of $4 \mathrm{G}$ networks: FDD or TDD. IEEE Commun. Mag. 44, 42-50 (2006)

5. ETSI. GSM recommendations, 04 May1993, Data Link Layer - General aspects (1993) 
6. 3GPP, TS 25 211, Physical Channels and Mapping of Transport Channels onto Physical Channels (FDD) (2001)

7. IEEE Std, I. 802.16-2004, IEEE Standard for Local, Metropolitan Area Networks, Part 16: Air Interface for Fixed Broadband Wireless Access Systems, October 2004

8. Pedersen, K.I., et al.: A flexible 5G frame structure design for frequency-division duplex cases. IEEE Comm. Mag. 54, 53-59 (2016)

9. Ramanathan, R.: On the performance of Ad hoc networks with beamforming antennas. In: Proceedings of ACM MobiHoc, pp. 95-105 (2001)

10. Jain, V., Gupta, A., Agrawal, D.P.: On-demand medium access in multihop wireless networks with multiple beam smart antennas. IEEE Trans. Parallel Distrib. Syst. 19(4), 489-502 (2008)

11. Feige, U., Kogan, S.: Hardness of approximation of the Balanced Complete Bipartite Subgraph problem. Department of Computer Science and Applied Mathematics, Weizmann Institute of Science, Rehovot, Israel, Technival Report MCS04 2004 (2004) 